\title{
Quality Control of Spirometry
}

Die retrospektive Analyse von Hagemeyer u. Mitarb. verdient Beachtung und zeugt von Mut, auch wissenschaftlich unbefriedigende Daten zu publizieren [1]. Sie zeigt im Wesentlichen die Unbrauchbarkeit von spirometrischen Größen, die von Ungeschulten mit nicht standardisierten Geräten und Messvorgängen in Unkenntnis der Leitlinien zur Qualitätssicherung erhoben wurden.

Wie könnte man in Zukunft die Qualität der spirometrisch fassbaren Größen verbessern und die Aussagekraft dieser Messparameter auf ein befriedigendes und in der Praxis brauchbares Niveau heben?

Dass nicht einmal 10\% der Geräte am Tag der Messung geeicht wurden, dürfte für die große Messwertstreuung und den ungenügenden Qualitätsscore von 0,58 bei maximal 1 noch den geringsten Einfluss gehabt haben. Abhilfe würde hier eventuell ein Computerprogramm schaffen, welches ohne den täglichen vorausgehenden Eichvorgang die spirometrische Messung nicht starten ließe. Da offensichtlich immer noch Geräte Verwendung finden und abrechnungsfähig sind, die nur Zahlen (sog. „Hausnummern“) der Messwerterhebung ohne zugehörige Analogkurven ausdrucken respektive anzeigen, ist sicher ein technischer Überwachungsdienst gefragt, solche Spirometer für die Krankenversorgung aus dem Verkehr zu ziehen.

Ein weiteres Problem zeigt diese Arbeit deutlich, nämlich die Sollwertsauswahl und Erhebung. Die Körpergröße und das Gewicht werden meist nicht gemessen, sondern nur erfragt. Die Sollwertskollektive sind meistens ungenügend auf „Normalität“ geprüft (Anamnese etc.), und die Frauen wurden nicht gemessen, sondern aus den Männerwerten mit einem Abzug von 10-15\% berechnet!
Jeder Arzt sollte daher mit seinem ihm bekannten „normalen“ Referenzkollektiv die Kompatibilität mit den von ihm verwendeten Sollwertskollektivformeln vergleichen, was keine große Stichprobe verlangt. Sollwerte für Raucher und Adipöse sind nicht notwendig, da es sich dabei bekanntlich um krankhafte Abweichungen handelt, die spirometrisch fassbar, aber nicht im vornherein zu berücksichtigen sind. Zu kleine Sollwerte sind für die Krankheitserfassung und für gutachterliche Stellungnahmen eine unfaire Benachteiligung des Patienten.

Auch die Reproduzierbarkeit der Messwerte kann am besten mit einem entsprechenden Computerprogramm unterstützt werden, zum Beispiel durch Messwertsausgabe erst nach mindestens drei Atemmanövern mit Angabe der Abweichung der besten

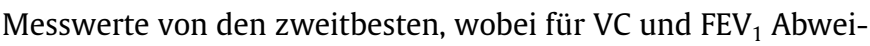
chungen von unter $5 \%$ angestrebt werden.

Ein fast weltanschauliches Problem ist, welches Atemmanöver für die spirometrischen Werterhebung das optimale sei. Wir sind der Meinung, dass von der Ruheatmung aus zuerst maximal exspiriert, dann inspiriert und anschließend forciert expiriert werden sollte. Dieser Ablauf des Atemmanövers muss der Patient auch bei vielen anderen Lungenfunktionstest (z.B. CO-Einatemzugdiffusionskapazitätsmessung) und therapeutischen sowie diagnostischen Aerosolinhalationen durchführen. Eine solche „unité de doctrine“ erlaubt eine schnellere und reproduzierbarere Erhebung der wenigen notwendigen Lungenfunktionsdaten, ohne viele Patienten durch Instruktion immer neuer und verschiedener Atemmanöver zu überfordern. Fällt so die inspiratorische Vitalkapazität (IVC) kleiner aus als die forciert expiratorisch gemessene (FVC) ist das Atemmanöver nicht optimal durchgeführt worden. Ist hingegen die FVC < IVC, besteht meist eine obstruktive Ventilationsstörung, welche durch den Tiffe- 
neau-Index $\left(\mathrm{FEV}_{1} / \mathrm{IVC}\right.$ resp. $\left.\mathrm{FEV}_{1} / \mathrm{FVC}<0,7\right)$ noch überprüft werden kann. Die rein forciert expiratorisch gemessene Spirometrie aus maximaler Inspirationslage mit mindestens 6 Sekunden Exspirationszeit ist in der Studie von Hagemeyer u. Mitarb. (ATSLeitlinien) einer der am wenigsten eingehaltenen Reproduktionskriterien. Die alleinige Messung des forcierten exspiratorischen Vitalkapazitätsmanövers hat in der Alltagspraxis erhebliche Nachteile, insbesondere bei der Interpretation obstruktiver Ventilationsstörungen, was mittlerweile auch von amerikanischen Autoren (Stoller u. Mitarb. 1997) an Patienten mit $\alpha 1$-Antitrypsinmangelemphysem erkannt und artikuliert wurde.

Moderne Spirometer erlauben daher nicht nur expiratorisch sondern auch inspiratorisch die Volumen-Zeit mit den zugehörigen Fluss-Volumenkurven zu registrieren. Letztere erlauben das bessere Erkennen extrapulmonaler Atemwegsobstruktionen. Die Kriterien für die Diagnose und Differenzialdiagnose extrapulmonaler Atemwegsobstruktionen mittels eines zusätzlichen forcierten inspiratorischen Atemmanövers werden in den meisten Leit- linien vernachlässigt. Auch bei Hagemeyer u. Mitarb. wird zwischen forcierter inspiratorischer und langsam inspiratorischer Vitalkapazität nicht unterschieden, wobei das Abkürzungssymbol IVC von ihnen ganz anders als allgemein üblich verwendet wird. Es ist zu hoffen, dass diese Arbeit nicht nur zu einer besseren Standardisierung der spirometrisch fassbaren Lungenvolumina mit zugehörigen Atemstromstärken führt, sondern auch zu einer besseren Messqualität mittels angepassten Computerprogrammen mit Reproduktions- und Abrechnungsdokumentation, sowie Ausbildungs- und Fortbildungsvorschriften. Nur dadurch kann die Qualität der ärztlichen Leistungen im pneumologischen, HNO- und arbeitsmedizinischen Funktionsbereich angehoben werden.

\section{Literatur}

${ }^{1}$ Hagemeyer O, Koppisch D, Otten H. Evaluation der Qualität von Spirometrien mittels Scores. Pneumologie 2004; 58: $147-154$ 